\title{
Effects of Platelet Agonists and Priming on the Formation of Platelet Populations
}

\author{
Alicia Veninga ${ }^{1}$ Constance C. F. M. J. Baaten ${ }^{1,2}$ Ilaria De Simone ${ }^{1,3}$ Bibian M. E. Tullemans ${ }^{1}$ \\ Marijke J. E. Kuijpers ${ }^{1,4}$ Johan W. M. Heemskerk ${ }^{1}$ () Paola E. J. van der Meijden $^{1,4}$ \\ ${ }^{1}$ Department of Biochemistry, Cardiovascular Research Institute \\ Maastricht, Maastricht University, The Netherlands \\ 2 Institute for Molecular Cardiovascular Research, University Hospital \\ Aachen, RWTH Aachen University, Germany \\ Address for correspondence Paola E. J. van der Meijden, PhD, \\ Department of Biochemistry, Cardiovascular Research Institute \\ Maastricht, Maastricht University, P.O. Box 616, 6200 MD, Maastricht, \\ The Netherlands (e-mail: p.vandermeijden@maastrichtuniversity.nl).
}

3 Institute for Cardiovascular and Metabolic Research, University of Reading, Reading, United Kingdom

${ }^{4}$ Thrombosis Expertise Center, Heart and Vascular Center, Maastricht University Medical Center, Maastricht, The Netherlands

\begin{abstract}
Keywords

- platelets

- populations

- priming

- activation markers

- flow cytometric analysis

Platelets from healthy donors display heterogeneity in responsiveness to agonists. The response thresholds of platelets are controlled by multiple bioactive molecules, acting as negatively or positively priming substances. Higher circulating levels of priming substances adenosine and succinate, as well as the occurrence of hypercoagulability, have been described for patients with ischaemic heart disease. Here, we present an improved methodology of flow cytometric analyses of platelet activation and the characterisation of platelet populations following activation and priming by automated clustering analysis.

Platelets were treated with adenosine, succinate, or coagulated plasma before stimulation with CRP-XL, 2-MeSADP, or TRAP6 and labelled for activated integrin $\alpha_{11 b} \beta_{3}$ (PAC1), CD62P, TLT1, CD63, and GPIX. The Super-Enhanced Dmax subtraction algorithm and $2 \%$ marker (quadrant) setting were applied to identify populations, which were further defined by state-of-the-art clustering techniques (tSNE, FlowSOM).

Following activation, five platelet populations were identified: resting, aggregating $(\mathrm{PAC} 1+$ ), secreting ( $\alpha$ - and dense-granules; CD62P,+ TLT1,$+ \mathrm{CD} 63+)$, aggregating plus $\alpha$-granule secreting (PAC1,$+ \mathrm{CD} 62 \mathrm{P}+, \mathrm{TLT} 1+$ ), and fully active platelet populations. The type of agonist determined the distribution of platelet populations. Adenosine in a dose-dependent way suppressed the fraction of fully activated platelets (TRAP6 $>$ 2-MeSADP $>$ CRP-XL), whereas succinate and coagulated plasma increased this fraction (CRP-XL $>$ TRAP6 $>2$-MeSADP). Interestingly, a subset of platelets showed a constant response (aggregating, secreting, or aggregating plus $\alpha$-granule secreting), which was hardly affected by the stimulus strength or priming substances.
\end{abstract}

received

December 14, 2020 accepted after revision July 12, 2021 published online October 24, 2021
DOI https://doi.org/ $10.1055 / \mathrm{s}-0041-1735972$. ISSN 0340-6245.

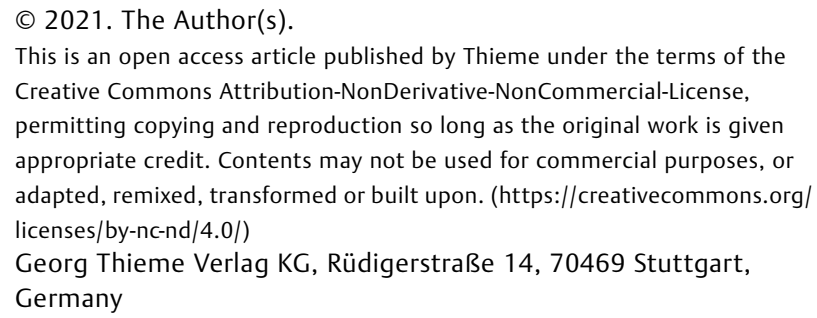




\section{Introduction}

Circulating platelets are heterogeneous in size, surface receptor expression levels, and age. This heterogeneity is related to differences in the platelet-producing megakaryocytes and to exposure to environmental changes during the platelet lifetime. ${ }^{1}$ As a consequence, individual platelets may respond differently to the same agonist, and may form populations with specific phenotypic characteristics and associated functions. 1,2

The properties of platelets, both in the circulation and during residence, e.g., in the spleen, are modulated by their continuous exposure to bioactive molecules. Such priming substances are considered to change the activation threshold of a platelet. Previously, we defined molecules that increase this threshold as negative primers, whereas positive primers decrease this threshold. ${ }^{1}$ In addition to the negatively priming platelet antagonists-prostacyclin, nitric oxide carriersand positively priming weak agonists-adrenaline, thrombopoietin, prostaglandins $s^{2,3}$-a large number of other priming substances in the blood will together set the thresholds of a platelet for activation. The overall equilibrium between negative and positive priming substances may shift in disease states, thus making circulating platelets more or less prone to activation. ${ }^{1}$

In patients with ischaemic heart disease, increased circulating levels of both adenosine and succinate have been described. ${ }^{4-7}$ Adenosine is released by myocardial, endothelial, and immune cells during conditions of ischaemia and inflammation, which occur in coronary artery disease. It is also generated in the extracellular space from adenosine triphosphate via ecto-nucleotidases, such as CD39 and CD73. ${ }^{8,9}$ In the cardiovascular system, adenosine exerts cardioprotective effects by, for example, increasing coronary blood flow and inhibiting platelet activation. ${ }^{10}$ In platelets, its binding to the Gs-protein-coupled $\mathrm{A}_{2 \mathrm{~A}}$ and $\mathrm{A}_{2 \mathrm{~B}}$ adenosine receptors results in cyclic adenosine monophosphate (cAMP) elevation and platelet suppression. ${ }^{11,12}$ While the normal adenosine level in blood is around $15 \mathrm{nM},{ }^{13}$ its level can greatly increase upon pathophysiological conditions. ${ }^{14,15}$ Succinate, a known player of intercellular communication, ${ }^{16}$ can also be released from ischaemic cardiomyocytes, as a consequence of mitochondrial dysfunction. ${ }^{17-19}$ Plasma levels of succinate can increase from approximately $5 \mu \mathrm{M}$ to millimolar levels during heavy exercise or in chronic disease conditions. ${ }^{20,21}$ Succinate acts on platelets via a Gi-proteincoupled receptor (SUCNR1, gene GPR91) and, likely by the lowering of cAMP, enhances platelet responses. ${ }^{22}$ Another factor associated with ischaemic heart disease that can affect platelet responsiveness is the hypercoagulable state of these patients. $^{23,24}$ Apart from the established platelet-activating role of thrombin, other coagulation-generated factors might alter platelet activation processes. ${ }^{25,26}$ It is still unclear how the priming substances in patients with ischaemic heart disease influence the activation profiles of platelets and the formation of distinct platelet populations.

Multicolour flow cytometry provides a sensitive method to distinguish and quantitate distinct subsets of monocytes,
T cells, and B cells. ${ }^{27,28}$ A few studies have used similar methods to also distinguish populations of activated platelets. ${ }^{29-31}$ However, when applying multicolour flow cytometry to samples of platelets, technical challenges arise for proper quantification. Per surface marker, (1) platelets may show an intermediate or high activation profile, (2) populations of resting and activated platelets may partially overlap, and (3) activation kinetics are fast and can be reversible, in contrast to the slow and persistent appearance of leukocyte subset differentiation markers. By implication, the normal use of a $2 \%$ marker set on negative samples ${ }^{32,33}$ may not give the best estimate of a certain (activated) platelet population, while the mean or median fluorescence intensity (MFI) provides no information on the fraction of cells with specific markers. As a better approach, flow cytometric software, such as FlowJo, provides improved mathematical procedures to estimate activation-induced shifts in different fluorescence histograms and to identify platelet populations.

In this article, by multicolour flow cytometry and the use of new mathematical tools, we investigated the effect of priming substances in blood plasma and serum on agonist-induced platelet activation profiles as well as on the formation of platelet populations. Our results revealed the importance of the agonist type compared with agonist dose and the presence of primers in the distribution of platelet populations.

\section{Methods}

\section{Materials}

Fluorescein isothiocyanate (FITC)-labelled PAC1 monoclonal antibody $(\mathrm{mAb})$ against activated human integrin $\alpha_{\mathrm{IIb}} \beta_{3}$, BV421-labelled anti-human Trem-like transcript 1 (TLT1) protein mAb, BV510-labelled anti-human CD42a mAb, and Cytofix were obtained from BD Bioscience (Franklin Lakes, New Jersey, United States), AF647-labelled and PerCP-Cy5.5labelled anti-human CD62P mAb were from Biolegend (San Diego, California, United States), and APC-labelled CD63 mAb was from Invitrogen, Fisher Scientific (Carlsbad, California, United States). Cross-linked collagen-related peptide (CRP$\mathrm{XL}$ ) was obtained from CambCol Laboratories (Cambridge, United Kingdom); 2-methylthio-adenosine-diphosphate (2MeSADP; stable ADP analogue) and D-phenylalanyl-prolylarginyl chloromethyl ketone (PPACK) from Santa Cruz Biotechnology (Dallas, Texas, United States), and thrombin receptor activating peptide 6 (TRAP6) from Bachem (Bubendorf, Switzerland). Succinic acid disodium salt (succinate), adenosine, and ellagic acid were purchased from SigmaAldrich (St. Louis, Missouri, United States). Recombinant human tissue factor was from Dade Behring (Breda, The Netherlands).

\section{Blood Donors}

According to the Helsinki Declaration, all blood donors gave written informed consent for participation. Studies were approved by the local medical ethics committee. Blood was collected from healthy volunteers (no antiplatelet medication for at least 2 weeks) from the antecubital vein into 3.2\% trisodium citrate tubes with a Vacutainer 21-gauge needle 
(Becton-Dickinson Bioscience). The first $5 \mathrm{~mL}$ of collected blood were discarded or collected into a serum tube.

\section{Preparation of Platelets}

Preparation of platelet-rich plasma (PRP) and platelets was essentially as described before. ${ }^{34}$ In brief, PRP was prepared by centrifugation at $260 \mathrm{~g}$ for 15 minutes of citrated blood. Where required, in PRP the platelet count was adjusted to $250 \times 10^{9} / \mathrm{L}$ by diluting with autologous platelet-free plasma. For preparation of washed platelets, PRP was supplemented with 1/10 acidic citrate dextrose (ACD; $80 \mathrm{mM}$ trisodium citrate, $52 \mathrm{mM}$ citric acid, and $180 \mathrm{mM}$ glucose) before centrifugation at $2,230 \mathrm{~g}$ for 2 minutes. The platelet pellet was resuspended in Hepes buffer pH 6.6 (10 mM Hepes, $136 \mathrm{mM} \mathrm{NaCl}, 2.7 \mathrm{mM} \mathrm{KCl}$, $2 \mathrm{mM} \mathrm{MgCl}_{2}, 0.1 \%$ glucose, and $0.1 \%$ bovine serum albumin, BSA). After supplementation with $1 / 15 \mathrm{ACD}$ and $1 \mathrm{U} / \mathrm{mL}$ apyrase, the cells were centrifuged at $2,230 \mathrm{~g}$ for 2 minutes. The platelets were then resuspended into Hepes buffer $\mathrm{pH} 7.45$ (10 mM Hepes, $136 \mathrm{mM} \mathrm{NaCl}, 2.7 \mathrm{mM} \mathrm{KCl}, 2 \mathrm{mM} \mathrm{MgCl} 2,0.1 \%$ glucose, and 0.1\% BSA). Platelet count was measured by Sysmex XP300 thrombocounter (Chuo-Ku, Kobe, Japan) and adjusted to $50 \times 10^{9} / \mathrm{L}^{29}$

\section{Preparation of Serum and Coagulated Plasma Samples} Serum was obtained from CT Serum Fast Separator tubes (Greiner Bio-One) and plasma from citrate tubes by centrifugation at 2,200 $\mathrm{g}$ for 10 minutes. Platelet-poor plasma (PPP) was obtained from PRP by a similar centrifugation step.

Where indicated, PRP and PPP were recalcified with $16.6 \mathrm{mM} \mathrm{CaCl}_{2}$ and stimulated with $25 \mu \mathrm{g} / \mathrm{mL}$ ellagic acid or $10 \mathrm{pM}$ tissue factor at $37^{\circ} \mathrm{C}$ for 1 hour, after which clots were manually removed. Sera, PRPs, and PPPs were centrifuged again after clot removal at 22,500 $\mathrm{g}$ for 5 minutes to remove all cell debris. Thrombin was inhibited by addition of $100 \mathrm{nM}$ PPACK. The sera from four healthy volunteers were pooled for experimentation.

\section{Platelet Activation and Flow Cytometry}

Washed platelets $\left(50 \times 10^{9} / \mathrm{L}\right)$ were preincubated with adenosine for 15 minutes, and with other priming substances immediately before agonist addition. The platelets were then stimulated for 15 minutes with indicated doses of CRP-XL (0.5-5 $\mu \mathrm{g} / \mathrm{mL})$, 2-MeSADP $(0.06-2 \mu \mathrm{M})$, or TRAP6 (2.5-15 $\mu \mathrm{M})$ in the presence of $2 \mathrm{mM} \mathrm{CaCl}$. All incubations were done at room temperature. Per blood donor, suboptimal agonist doses were preset by dose finding using nonprimed platelets to obtain 40 to $60 \%$ platelets with integrin $\alpha_{\mathrm{IIb}} \beta_{3}$ activation applying the $2 \%$ marker setting. Directly after addition of the agonist, the platelets were stained for activated integrin $\alpha_{\mathrm{II}} \beta_{3}$ and P-selectin expression using FITCconjugated PAC1 mAb $(1.25 \mu \mathrm{g} / \mathrm{mL})$ and AF647-conjugated anti-P-selectin $\mathrm{mAb}(2.5 \mu \mathrm{g} / \mathrm{mL})$, respectively. The activation studies were performed at least in duplicate. Per sample, 5,000 events were measured using a BD Accuri C6 flow cytometer (BD Bioscience, Franklin Lakes, New Jersey, United States). ${ }^{29}$

For the multicolour experimentation, washed platelets $\left(25 \times 10^{9} / \mathrm{L}\right)$ were stimulated with indicated doses of either
CRP-XL $(0.5-5 \mu \mathrm{g} / \mathrm{mL}), 2$-MeSADP $(0.06-2 \mu \mathrm{M})$, or TRAP6 $(2.5-15 \mu \mathrm{M})$ in the presence of $2 \mathrm{mM} \mathrm{CaCl}_{2}$ and stained with BV421-labelled anti-TLT1 mAb $(4 \mu \mathrm{g} / \mathrm{mL})$, BV510-labelled anti-CD42a mAb (4 $\mu \mathrm{g} / \mathrm{mL})$, FITC-labelled PAC1 mAb (1.25 $\mu \mathrm{g} / \mathrm{mL})$, PerCP-Cy5.5-labelled anti-CD62P mAb $(0.2 \mu \mathrm{g} / \mathrm{mL})$, and APC-labelled anti-CD63 mAb (1:20). Platelets were activated and stained for 15 minutes, after which $0.3 \%$ Cytofix was added. Samples were fixated for at least 30 minutes at $4^{\circ}$ C in the dark before measuring 10,000 events on the BD FACSCanto II (BD Bioscience, Franklin Lakes, New Jersey, United States) per sample.

\section{Analysis of Flow Cytometric Data}

Flow cytometric data were analysed using FlowJo V10 software (TreeStar, Ashland, Oregon, United States). Conventionally, positive events were set by thresholding at $2 \%$ of the unstimulated sample as positive, after gating for single cells. Therefore, markers were entered into two-colour dot plots by using the command Make Quad Gates, at 98 th percentiles ( $2 \%$ marker setting). In addition, percentages of positive platelets per fluorescence channel were calculated with the comparison algorithm Super-Enhanced Dmax (SED) subtraction. ${ }^{35}$ The SED algorithm is based on subtractions of a control histogram, taking into account the overall event distribution and the outlier events. Positive events determined by SED were obtained by the Population Comparison command, after importing datasets from concatenated duplicate or triplicate control samples, from which differences were calculated in comparison to the control sample.

For analysis of multicolour flow cytometric data, the separate FCS files were opened in FlowJo and checked for data anomalies by FlowAI. ${ }^{36}$ Before concatenating the samples that were compared, the event count per sample was set at 5,000 by DownSample. The samples were gated for single cells and the presence of CD42a. After concatenation, the resulting file was subjected to FlowSOM analysis ${ }^{37}$ for creating five clusters. FlowSOM performs a two-stage clustering. First, all data are put onto an input matrix (default $10 \times 10$ grid) on which a self-organising map (SOM) algorithm is trained to assign data points in the dataset to the node they most resemble, resulting in 100 little clusters in default settings. Subsequently, the data are subjected to metaclustering, a second stage of clustering of these 100 clusters into the final populations. This unsupervised analysis minimises the risk of missing populations and gives a clear overview of the clusters found. Detailed information on FlowSOM is described previously. ${ }^{37}$ By gating on the SampleID parameter, the individual sample conditions were rediscovered in the concatenated file and the fractions of platelets present in the resulting FlowSOM populations were determined. To visualise these high-dimensional multicolour flow cytometric data in a two-dimensional plot, t-distributed stochastic neighbor embedding (tSNE) analysis was applied.

\section{Statistical Analysis}

Significances of differences in frequencies of platelet populations between sample conditions were tested by chisquare with multiple comparisons tests with false discovery 
rate correction. Comparison of analysis methods ( $2 \%$ marker setting vs. SED algorithm) were done by using the MannWhitney U test.

$p$-Values $<0.05$ were considered as significant. Statistical analysis (chi-square test) was performed using R (Vienna, Austria), whereas other statistical analyses were done, and graphs were made using GraphPad Prism 7.00 (GraphPad Software, San Diego, California, United States).

\section{Results}

Novel Analytical Gating Strategy to Determine Platelet Activation Fractions by Flow Cytometry

In flow cytometric evaluation of platelet activation, the conventional use of a $2 \%$ positive threshold marker has limitations. These arise from low fluorescence shifts of activation markers with low copy numbers and from incomplete shifts seen with low agonist doses. Accordingly, in platelets stimulated with suboptimal doses of CRP-XL, 2MeSADP, or TRAP6, the flow cytometric histogram of integrin $\alpha_{\text {IIb }} \beta_{3}$ activation (FITC-PAC1 $\mathrm{mAb}$ ) showed only a partial right shift ( - Fig. 1A). Similarly, these suboptimal doses provoked no more than small histogram changes for secretion, assessed with an $\alpha$-granule secretion marker (AF647 $\alpha$-Pselectin $\mathrm{mAb}$ ) ( - Fig. 1B). Furthermore, the MFI indicates the shift in fluorescence intensity of a population of platelets, but lacks information on the shape of the histogram, which often not follows a normal distribution with suboptimal doses of agonists, and on the fraction of platelets with activation markers. Markedly, superimposed two-colour dot plots suggested the appearance of a distinguishable platelet fraction upon stimulation with the suboptimal agonist concentrations (-Fig. 1C, assigned in blue). Advanced histogram analysis is needed to quantify this fraction.

As a sensitive mathematical tool to evaluate partial curve shifts, we used the algorithm SED subtraction in FlowJo V10. This algorithm subtracts the histogram of concatenated control samples from the histograms of interest of activated platelets, taking into account the data distribution profile and outlier events. When applied to a suboptimal dose of 2MeSADP, SED calculated a fraction of $64 \%$ platelets with increased PAC1 staining, i.e., much higher than the fraction of $50 \% \mathrm{Psel}^{+}$platelets obtained with the $2 \%$ threshold marker setting (-Fig. 1D). When extended to all agonist conditions, the combination of the two analytical tools gave complementary pieces of information on: (1) a quantified fraction of platelets with any activated integrin $\alpha_{\mathrm{IIb}} \beta_{3}$ (SED algorithm), and (2) the fraction of platelets with $>98 \%$ probability of integrin activation. Calculations learned that the SED subtraction method gave $+7-20 \%$ higher percentages of (intermediately) activated platelets showing the respective activation markers (-Table $\mathbf{1}$ ).

We further considered that the SED subtraction may be the most sensitive to detect a negative or positive priming state of platelets, but this method can only be used for the analysis of single fluorescence labels. The $2 \%$ threshold setting will identify platelets with ensured integrin $\alpha_{\mathrm{IIb}} \beta_{3}$ activation and/or P-selectin expression. It should be taken
A

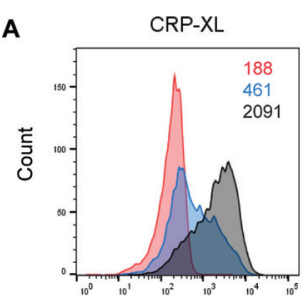

2-MeSADP
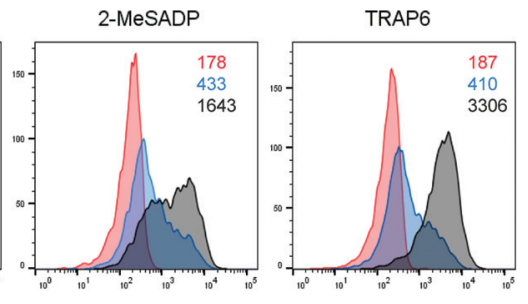

B

FITC-PAC1
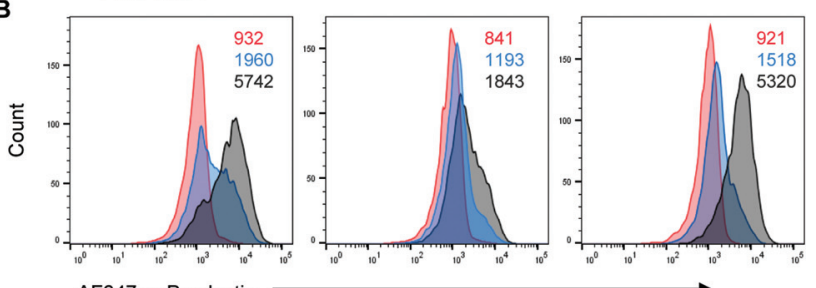

C

AF647- a P-selectin
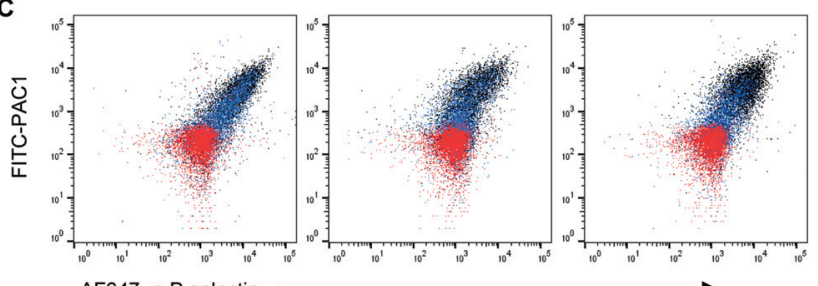

D

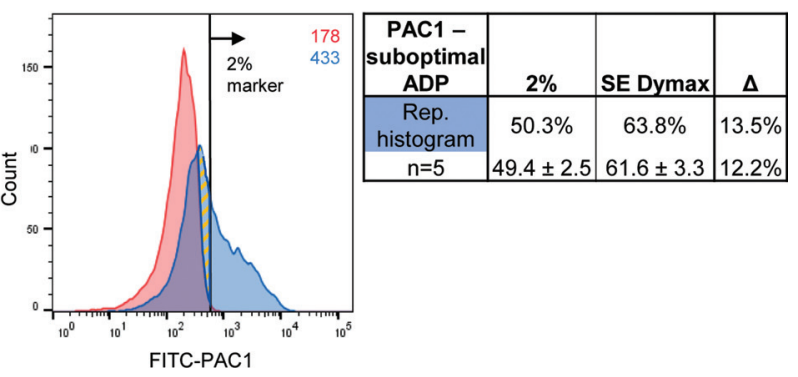

Fig. 1 Effect of agonist dose on histogram changes of platelet activation markers. Washed platelets were stimulated with a suboptimal or optimal dose of CRP-XL, 2-MeSADP, or TRAP6, established per donor, for 15 minutes. After stimulation, the cells were labelled with AF647 anti-P-selectin mAb (secretion marker) and FITC-PAC1 mAb (integrin $\alpha_{11 b} \beta_{3}$ activation marker) and analysed by multicolour flow cytometry. Shown are representative histograms and fluorescence dot plots $(n=5)$ per agonist; CRP-XL $(0.5 \mu \mathrm{g} / \mathrm{mL} ; 5 \mu \mathrm{g} / \mathrm{mL})$, 2-MeSADP (25 nM; $2 \mu \mathrm{M})$, or TRAP6 $(7.5 \mu \mathrm{M} ; 15 \mu \mathrm{M})$, including the median fluorescence intensity (MFI). Colour code representing different samples: unstimulated platelets (red), platelets stimulated with suboptimal agonist dose (blue), and platelet stimulated with optimal agonist dose (black). (A) Staining with FITC-PAC1 mAb, (B) AF647 $\alpha$-Pselectin mAb staining, (C) dual-colour dot plots. (D) Representative flow cytometric histograms of PAC1 staining of unstimulated platelets (red) and platelets stimulated with suboptimal dose of $0.1 \mu \mathrm{M} 2$ MeSADP (total blue area). Indicated are platelets positive by $2 \%$ marker setting (right of vertical line) as well as the positive platelet fraction calculated by Super-Enhanced Dmax (SED) subtraction. Yellow-striped area represents area difference between the two settings. The table depicts population of $\mathrm{PAC}^{+}$platelets according to $2 \%$ marker setting or to SED analysis. Representative for $n=5$ platelet samples (6$250 \mathrm{nM}$ 2-MeSADP for suboptimal stimulation). mAb, monoclonal antibody.

into account that the $2 \%$ marker setting likely results in an underrepresentation of the platelets with any integrin activation or P-selectin expression. In the remaining part of this article, using two-colour flow cytometry, the $2 \%$ marker 
Table 1 Comparison of fractions of activated platelets by conventional 2\% threshold marker setting or by SED analysis

\begin{tabular}{|c|c|c|c|c|c|c|c|c|c|}
\hline \multirow[t]{2}{*}{ PAC1 } & \multicolumn{3}{|c|}{ CRP-XL } & \multicolumn{3}{|c|}{ 2-MeSADP } & \multicolumn{3}{|c|}{ TRAP6 } \\
\hline & $2 \%(\%)$ & SE Dymax (\%) & $p$-Value & $2 \%(\%)$ & SE Dymax (\%) & $p$-Value & $2 \%(\%)$ & SE Dymax (\%) & $p$-Value \\
\hline Unstimulated & $3.06 \pm 0.39$ & $2.98 \pm 2.15$ & 0.802 & $2.9 \pm 0.59$ & $3.03 \pm 1.86$ & 1.000 & $3.31 \pm 1.33$ & $4.63 \pm 3.91$ & 0.151 \\
\hline Intermediate & $48.86 \pm 5.34$ & $55.54 \pm 5.75$ & 0.056 & $49.44 \pm 2.48$ & $61.6 \pm 3.25$ & 0.008 & $53.17 \pm 8.74$ & $61.79 \pm 5.45$ & 0.103 \\
\hline Maximum & $85.74 \pm 5.42$ & $87.79 \pm 4.31$ & 0.421 & $66.91 \pm 11.04$ & \multicolumn{2}{|l|}{$75.49 \pm 8.66$} & $88.95 \pm 5.32$ & $91.26 \pm 3.85$ & 0.548 \\
\hline P-selectin & \multicolumn{3}{|c|}{ CRP-XL } & \multicolumn{3}{|c|}{ 2-MeSADP } & \multicolumn{3}{|c|}{ TRAP6 } \\
\hline & $2 \%(\%)$ & SE Dymax (\%) & $p$-Value & $2 \%(\%)$ & SE Dymax (\%) & $p$-Value & \multicolumn{3}{|c|}{ SF Dymay $1 \%$} \\
\hline Unstimulated & $3.29 \pm 0.28$ & $3.73 \pm 3.28$ & 0.691 & $3.87 \pm 1.32$ & $3.33 \pm 3.89$ & 0.548 & $3.46 \pm 0.67$ & $6.65 \pm 6.22$ & 0.008 \\
\hline Intermediate & $34.51 \pm 12.67$ & $45.02 \pm 14.08$ & 0.310 & $16.65 \pm 5.69$ & $36.80 \pm 6.21$ & 0.008 & $43.07 \pm 10.41$ & $55.64 \pm 6.95$ & 0.056 \\
\hline Maximum & $73.36 \pm 5.19$ & $81.71 \pm 3.95$ & 0.016 & $29.49 \pm 15.28$ & $52.65 \pm 6.95$ & 0.016 & $83.55 \pm 4.36$ & $88.55 \pm 2.97$ & 0.032 \\
\hline MFI & \multicolumn{3}{|c|}{ PAC1 } & \multicolumn{3}{|c|}{ P-selectin } & & & \\
\hline & CRP-XL & 2-MeSADP & TRAP6 & CRP-XL & 2-MeSADP & TRAP6 & & & \\
\hline Unstimulated & $193 \pm 8$ & $183 \pm 4$ & $184 \pm 13$ & $950 \pm 37$ & $984 \pm 76$ & $879 \pm 144$ & & & \\
\hline Intermediate & $458 \pm 141$ & $417 \pm 30$ & $459 \pm 167$ & $1,668 \pm 347$ & $1,377 \pm 173$ & $1,822 \pm 419$ & & & \\
\hline Maximum & $3,063 \pm 1,260$ & $924 \pm 453$ & $2,231 \pm 867$ & $5,896 \pm 1,028$ & $1,761 \pm 105$ & $5,717 \pm 727$ & & & \\
\hline
\end{tabular}

Abbreviations: MFI, median fluorescence intensity; SED, Super-Enhanced Dmax.

Note: Shown are percentages of assigned PAC1- or P-selectin-positive platelets, obtained by a $2 \%$ marker setting or SED analysis, for unstimulated samples or samples stimulated with suboptimal or maximum doses of CRP-XL, 2-MeSADP, or TRAP6. As well as the median fluorescence intensity $(\mathrm{MFI})$ values per condition. Mean $\pm \mathrm{SD}, n=5$, differences between positive platelets calculated by $2 \%$ marker setting and SED analysis calculated by the Mann-Whitney U-test. Statistically significant p-values are denoted in bold.

setting was used to distinguish the fractions of defined activated platelets.

\section{Platelets Vary in Agonist-Induced Responses of Integrin Activation and Secretion}

To assess the aggregating and secreting platelet populations, we generated dose-response information using on the $2 \%$ marker setting on the fractions of platelets with defined integrin activation $\left(\mathrm{PAC}^{+}\right)$and/or P-selectin expression $\left(\mathrm{Psel}^{+}\right)$. For two-colour dot plots of resting platelets (-Fig. 2A) and platelets stimulated with CRP-XL, 2-MeSADP, or TRAP6, the cross-marker setting thus resulted in quadrants, showing four platelet fractions, which for convenience were categorised as: $\mathrm{PAC1}^{+} / \mathrm{Psel}^{-}, \mathrm{PAC1}^{+} / \mathrm{Psel}^{+}, \mathrm{PAC1}^{-} / \mathrm{Psel}^{+}$, and $\mathrm{PAC1}^{-} / \mathrm{Psel}^{-}$(-Fig. 2B). While the PAC1 ${ }^{-}$and $\mathrm{Psel}^{-}$ fractions also included platelets with intermediate activation profiles, this two-colour analysis revealed typical differences between agonists.

For CRP-XL, the fractions of activated integrin only, 10$20 \% \mathrm{PAC}^{+} / \mathrm{Psel}^{-}$, and of secretion only, $1-5 \% \mathrm{PAC}^{-} / \mathrm{Psel}^{+}$, did not significantly change with a higher CRP-XL concentration (-Fig. 2C). These results imply the presence of a platelet population that is more sensitive to CRP-XL-induced integrin activation than to secretion. The doublepositive fraction gradually increased to approximately $80 \%$ at the highest agonist dose. Even at the highest CRP-XL dose, approximately $5 \%$ of the platelets appeared to be unresponsive.

Platelet stimulation with stable ADP also resulted in pronounced heterogeneity, this time with hardly any platelets showing only secretion (-Fig. 2D). Of all PAC1 ${ }^{+}$platelets, a consistent fraction of about one-third was double positive for all ADP doses. As expected, with this weak agonist an appreciable platelet population was more sensitive to integrin activation than to secretion. Platelet stimulation with TRAP6 resulted in a dose-dependent increase of the doublepositive population (-Fig. 2E). In addition, TRAP6 stimulation gave stable fractions of approximately $20 \% \mathrm{PAC}^{+} / \mathrm{Psel}^{-}$ platelets and 5\% $\mathrm{PAC1}^{-} / \mathrm{Psel}^{+}$platelets. Hence, upon stimulation of the thrombin receptor PAR1, sets of platelets only responded by integrin activation or secretion. None of the agonists tested induced a significant dose-dependent change in secretion- or aggregation-only populations. Both with stable ADP and TRAP6, residual fractions of platelets appeared to be unresponsive $\left(\mathrm{PAC}^{-} / \mathrm{Psel}^{-}\right.$), according to the $2 \%$ marker analysis.

Next, we checked whether these results were supported by automated clustering tools. Therefore, multicolour flow cytometry was used with antibodies against GPIX (CD42a) as a platelet surface marker, activated integrin (PAC1), P-selectin (CD62P), TLT1 as an alternative $\alpha$-granule secretion marker, and CD63 as a dense granule and lysosomal secretion marker. Since the $2 \%$ marker setting is not optimal for the identification of distinct populations and has limitations in multicolour settings, we applied a workflow in FlowJo consisting of FlowAI, DownSample, FlowSOM, and tSNE. FlowAI was used to clean the data, after which DownSample was used to subset the samples in a specified event count before concatenation. By using FlowSOM, a state-of-the-art clustering technique using SOMs, we obtained five different clusters/platelet populations and their abundancy in the samples. The tSNE algorithm was used to perform dimensionality reduction, allowing for visualisation of highdimensional data in a two-dimensional plot, with overlaying FlowSOM filter. Analysis of unstimulated platelets and maximal stimulated platelets with CRP-XL, stable ADP, or TRAP6 revealed five distinct populations. Differences in population distributions could be clearly observed in the tSNE plots with FlowSOM filter (-Fig. $\mathbf{3 A}$ ) and were characterised by the expression level of each marker ( - Fig. $\mathbf{3 B}$ ). This resulted in populations consisting of resting platelets (Pop0, $\mathrm{PAC1}^{-} /$ Psel $\left.^{-} / \mathrm{CD}^{-} 3^{-} / \mathrm{TLT}^{-} / \mathrm{GPIX}^{+}\right)$, platelets with only activated integrin (Pop1, $\mathrm{PAC}^{+} / \mathrm{Psel}^{-} / \mathrm{CD}^{-} 3^{-} / \mathrm{TLT}^{-} / \mathrm{GPIX}^{+}$), platelets with activated integrins and $\alpha$-granule secretion (Pop2, $\left.\mathrm{PAC}^{+} / \mathrm{Psel}^{+} / \mathrm{CD}^{-} 3^{-} / \mathrm{TLT}^{+} / \mathrm{GPIX}^{+}\right)$, platelets with only $\alpha-$ and dense granule/lysosome secretion (Pop3, $\mathrm{PAC}^{-} / \mathrm{Psel}^{+} /$ 
A

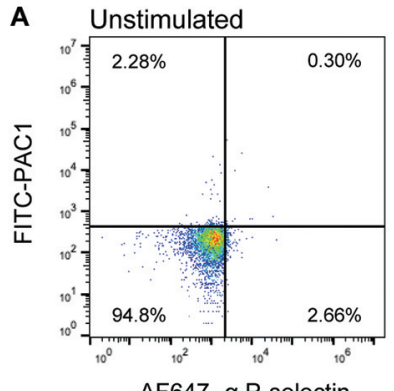

B Suboptimal TRAP6

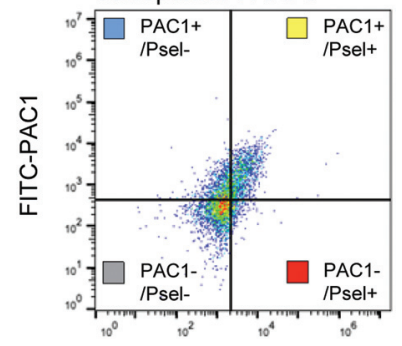

AF647- $\alpha$ P-selectin
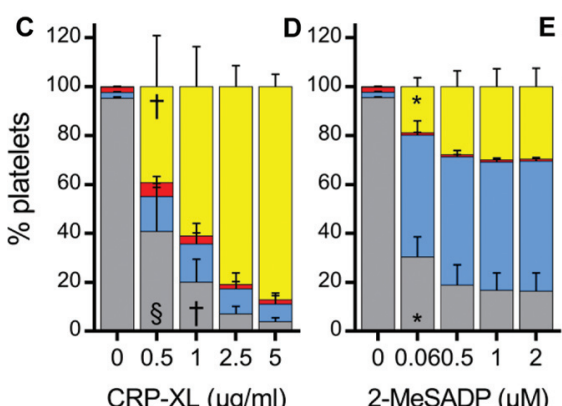

$E_{120}$

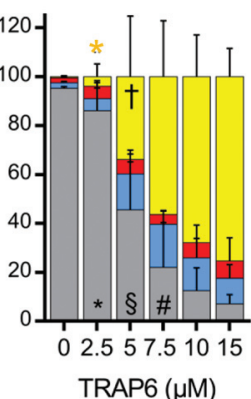

Fig. 2 Agonist- and dose-dependent formation of platelet populations with differential integrin activation and/or secretion. Washed platelets were stimulated with indicated concentrations of CRP-XL $(0.5-5 \mu \mathrm{g} / \mathrm{mL})$, 2-MeSADP $(0.06-2 \mu \mathrm{M})$, or TRAP6 $(2.5-15 \mu \mathrm{M})$, and then labelled and analysed as for $\boldsymbol{-}$ Fig. 1. Fluorescence thresholds in quadrant were set for unstimulated sample ( $2 \%$ positive events) per label. (A) Representative dot plot of unstimulated sample with $2 \%$ marker setting. (B) Platelet populations in response to $7.5 \mu \mathrm{M}$ TRAP6 were (clockwise): $\mathrm{PAC1}^{-} / \mathrm{Psel}^{-}$(gray), $\mathrm{PAC1}^{+} / \mathrm{Psel}^{-}$(blue), $\mathrm{PAC1}^{+} /$ $\mathrm{Psel}^{+}$(yellow), and PAC1 ${ }^{-} / \mathrm{Psel}^{+}$(red). (C-E) Mean fractions of different platelet populations as a function of indicated agonist and dose. Bar colours correspond to colour code in quadrant plot. Mean + SD, $n=4-5$, (C) ${ }^{\dagger} p<0.05$ compared with 2.5 and $5 \mu \mathrm{g} / \mathrm{mL}$ CRP-XL, ${ }^{\S} p<0.05$ compared with $1,2.5$, and $5 \mu \mathrm{g} / \mathrm{mL}$ CRP-XL; (D) ${ }^{*} p<0.05$ compared with all other conditions; (E) ${ }^{*} p<0.05$ compared with all other conditions, ${ }^{\S} p<0.05$ compared with $7.5,10$, and $15 \mu \mathrm{M}$ TRAP6, ${ }^{\dagger} p<0.05$ compared with 10 and $15 \mu \mathrm{M}$, ${ }^{\#} p<0.05$ compared with 15 $\mu \mathrm{M}$ TRAP6; changes in population frequencies between conditions tested by chi-square multi-comparison test with false discovery rate correction.

${\mathrm{CD} 63^{+}}^{+} \mathrm{TLT}^{+} / \mathrm{GPIX}^{+}$), and fully activated platelets with activated integrins and $\alpha$ - and dense-granule/lysosome secretion (Pop4, $\mathrm{PAC}^{+} / \mathrm{Psel}^{+} / \mathrm{CD} 3^{+} / \mathrm{TLT}^{+} / \mathrm{GPIX}^{+}$). Maximal stimulation with either CRP-XL or TRAP6 resulted in a similar distribution of the populations (not significantly different); $60-65 \%$ of events positive for all markers (Pop4), 5-10\% aggregating-only platelets (Pop1), 5-10\% secreting-only platelets (Pop3), $10-15 \%$ aggregating and $\alpha$-granule secreting platelets (Pop2), and $10-15 \%$ resting platelets (Pop0) (-Fig. 3C). Maximal stimulation with 2-MeSADP resulted in significantly different proportions of resting (Pop0), aggregating (Pop1), aggregating plus $\alpha$-granule secreting (Pop2), and completely activated (Pop4) platelet populations, compared with CRP-XL/TRAP6. Surprisingly, the fraction of secreting-only (Pop3) platelets was not significantly different. These analyses show that, regardless of agonist type and donor, there is a consistent population of platelets present with secreted granular content, but without integrin activation.

\section{Priming with Adenosine or Succinate Modulates the Resting and Fully Activated Platelet Populations}

To investigate how negative platelet priming affects the dual response heterogeneity, we used adenosine, which acts by raising the cAMP level. Platelets were pre-treated with adenosine, and subsequently stimulated with a suboptimal concentration of CRP-XL, 2-MeSADP, or TRAP6. We chose to use suboptimal doses of agonists to allow detection of enhanced (positive priming) and diminished (negative priming) platelet activation. Two-colour flow cytometric analysis indicated that adenosine priming reduced the number of platelets with both integrin $\alpha_{\mathrm{II}} \beta_{3}$ activation and P-selectin expression (-Fig. 4A). Quantification of populations using the $2 \%$ marker setting indicated with adenosine a dosedependent increase of the double negative fraction at the expense of the double positive fraction ( - Fig. 4B). The adenosine effect was more apparent after stimulation with 2-MeSADP or TRAP6 than with CRP-XL. In addition, with all three agonists, adenosine tended to decrease the fraction of $\mathrm{PACl}^{+} / \mathrm{Psel}^{-}$platelets (however only significant for 2MeSADP), while leaving the small $\mathrm{PAC1}^{-} / \mathrm{Psel}^{+}$fraction unchanged. Extending the data by multicolour flow cytometry and clustering analyses, confirmed that adenosine only significantly changed the resting (Pop0) and fully activated (Pop4) populations (- Fig. 4C-E). The inhibiting effect of adenosine $(1 \mu \mathrm{M})$ was significantly higher for TRAP6 and ADP compared with CRP-XL, based on the fully activated (Pop4) platelet population (p-values 0.011 and 0.004 , respectively). Apparently, a consistent fraction of platelets seemed to respond to the agonists in their specialised manner (Pop1, 2 and 3), regardless of the presence of adenosine.

Subsequently, we investigated the positive priming agent succinate, acting by cAMP lowering. Two-colour plots and $2 \%$ marker analysis demonstrated that succinate in particular increased the dual positive platelet fraction in response to CRP-XL or TRAP6, while the single positive $\mathrm{PAC1}^{+}$fraction in particular remained high with stable ADP stimulation ( -Fig. 5A, B). In this analysis, the population profile of ADP-stimulated platelets was hardly influenced by succinate priming. The multicolour flow cytometry results corroborate these findings. Succinate-induced significant changes in the fractions of resting (Pop0) and fully activated (Pop4) platelets (-Fig. 5C-E). Taken together, these results show that priming, in terms of changes in cAMP, most profoundly modulates the formation of resting and fully activated platelets, leaving the aggregation-only, secretion-only, and aggregation plus $\alpha$-granule secretion platelet populations relatively unchanged.

\section{Priming Effect on Platelet Populations by Serum and Coagulated Plasma}

Given the expected accumulation of platelet priming factors under coagulated conditions, we prepared samples of serum derived from whole blood or plasma. For the plasma samples, PRP and PPP were recalcified in the presence of ellagic acid (triggering the intrinsic coagulation pathway) or tissue factor (triggering the extrinsic pathway). Clots were removed from all samples, and the degradable thrombin active-site 
A
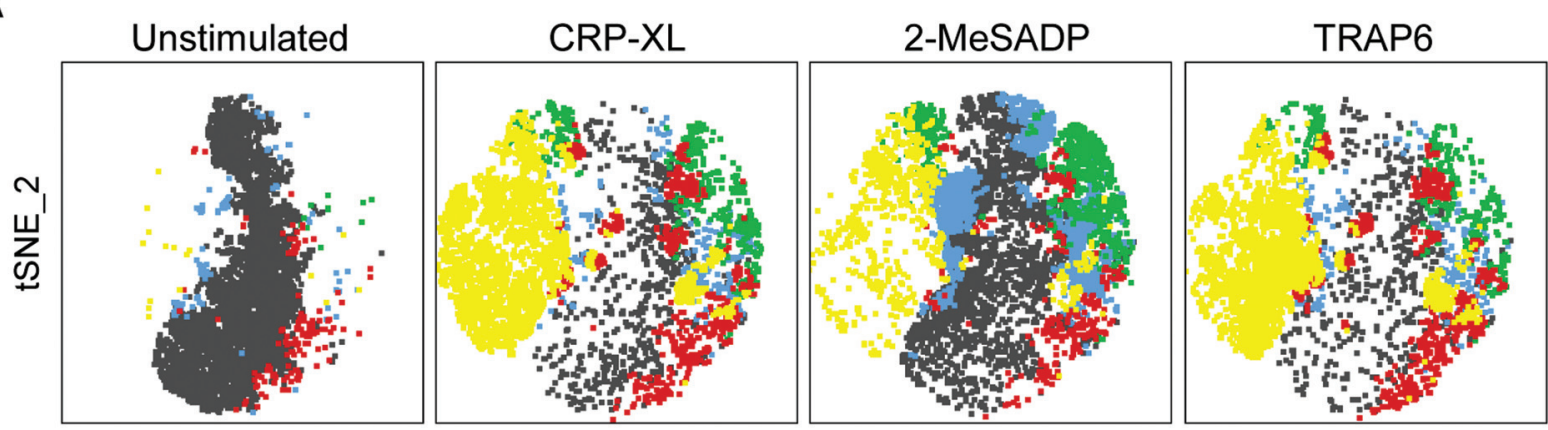

tSNE_1

B

\begin{tabular}{|l|c|c|c|c|c|}
\hline & PAC1 & CD62P & CD63 & TLT1 & GPIX \\
\hline Pop 0 & -- & -- & - & -- & + \\
Pop 1 & + & - & - & - & + \\
Pop 2 & + & + & -- & + & + \\
Pop 3 & - & + & + & + & + \\
Pop 4 & ++ & ++ & ++ & ++ & + \\
\hline
\end{tabular}

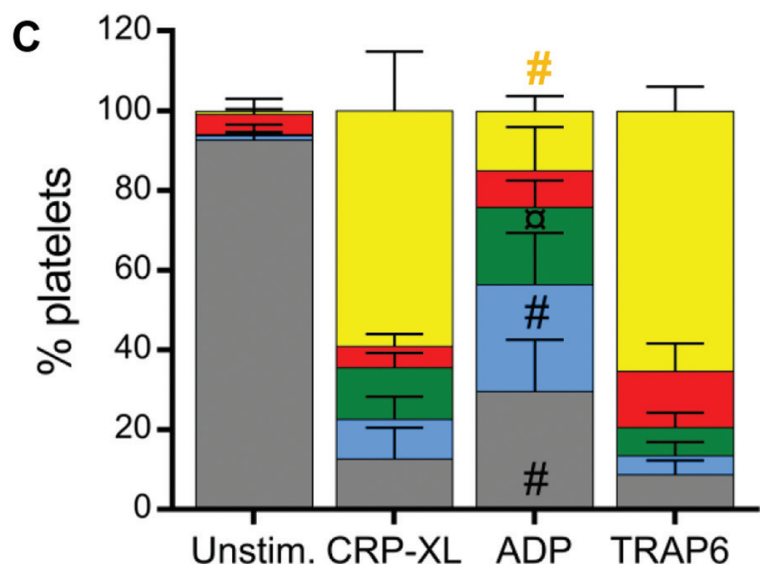

Fig. 3 Analysis of agonist-induced platelet populations by dimensionality reduction and clustering algorithms. Washed platelets were maximally stimulated with $5 \mu \mathrm{g} / \mathrm{mL}$ CRP-XL, $2 \mu \mathrm{M}$ 2-MeSADP, or $15 \mu \mathrm{M}$ TRAP6 followed by labelling with coloured PAC1, anti-CD62P, anti-CD63, anti-TLT1, and anti-GPIX mAbs for multicolour flow cytometry. (A) Representative tSNE plots of unstimulated, CRP-XL-, stable ADP- and TRAP6stimulated samples. (B) Characterisation of the FlowSOM clusters (5 populations). - /+ indicates within \pm 1 SD from the mean population MFI,-/ ++ indicates within \pm 2 SD from the mean population MFI. (C) Stacked bar graph of the percentage platelets per cluster/population. Mean + SD, $n=5$, ${ }^{*} p<0.05$ compared with CRP-XL and TRAP6, ap $<0.05$ compared with TRAP6; changes in population frequencies between conditions tested by chi-square multi-comparison test with false discovery rate correction. $\mathrm{mAb}$, monoclonal antibody; $\mathrm{MFI}$, median Fluorescence intensity; SD, standard deviation; tSNE, t-distributed stochastic neighbor embedding.

inhibitor PPACK was post-added to inactivate all thrombin. All samples were checked for the absence of residual factor Xa and thrombin activity (data not shown).

After exposure of the platelets to (whole blood) serum, the platelets were again activated with a suboptimal dose of CRP-XL, 2-MeSADP, or TRAP6. Two-colour plots showed a marked increase of the $\mathrm{PAC}^{+} / \mathrm{Psel}^{+}$platelets upon priming by serum (-Fig. 6A). This was confirmed by quantification of the platelet fractions, i.e., with all agonists, a serum-induced increase in the fraction of $\mathrm{PAC}^{+} /$ $\mathrm{Psel}^{+}$platelets, but an unchanged fraction of $\mathrm{PAC}^{+} / \mathrm{Psel}^{-}$ platelets (-Fig. 6B). The fraction of secretion-only platelets in this case remained negligible for the two-colour flow cytometric analysis. In contrast, automated clustering of the multicolour flow cytometric data revealed a consistent fraction of secreting-only platelets (Pop3; - Fig. 6C-E). The enhancing effect of serum on fully activated platelets was less pronounced with TRAP6 stimulation $(+7 \%)$, compared with ADP $(+21 \%)$ or CRP-XL ( $+19 \%)$. To investigate whether the positive priming found with serum originated from plasma or cellular components, the platelets were also preincubated with serum derived from coagulated PRP or
PPP. For all agonists, this resulted in the same potentiating effect as seen with serum, without causing activation in the absence of an agonist (-Fig. 6B, D). Noncoagulated normal pooled plasma did not enhance platelet activation in response to intermediate doses of agonists (-Supplemental Fig. $\mathbf{S 1}$ [available in the online version]). Together, this confirmed that compounds generated during the coagulation process are able to enhance platelet activation and predominantly increase the population of fully activated platelets.

\section{Discussion}

In this article, we used an optimised multicolour flow cytometric analysis workflow that allows for the automated identification of the populations of activated platelets. These data were compared with the more standard analysis method of applying the $2 \%$ marker setting. In addition, we set to disclose how positive or negative priming of the platelets led to changes in the populations. In general, it appeared that primarily the agonist type was determinative for the typical distribution of the different populations, 
A

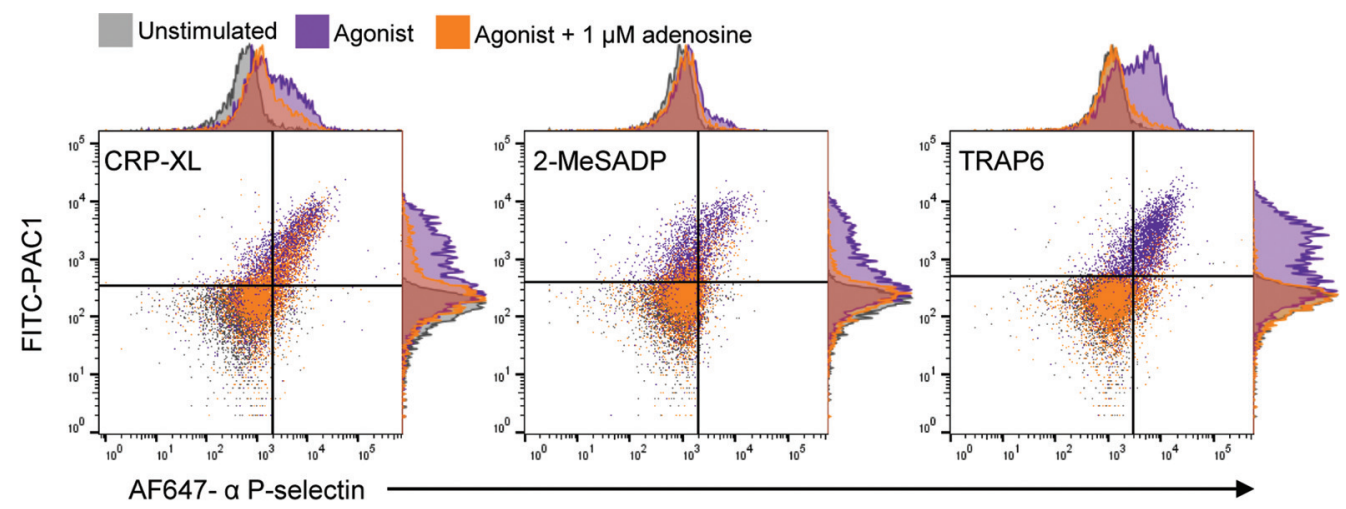

B PAC1-/Psel- $\quad$ PAC1+/Psel- $\quad$ PAC1-/Psel+ PAC1+/Psel+

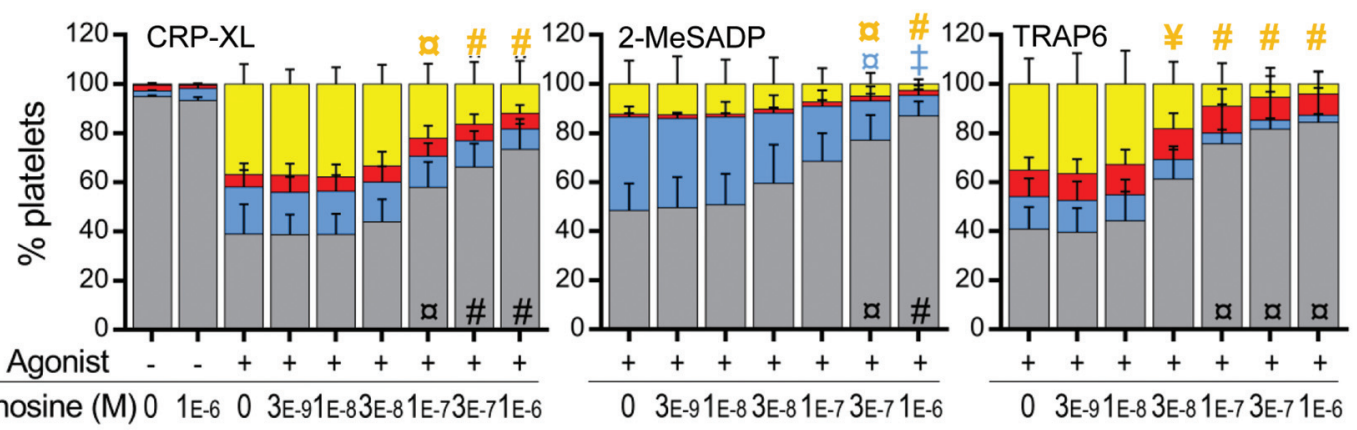

Adenosine (M) 0 1E-6 0 3E-91E-83E-81E-73E-71E-6

0 3E-91E-8 3E-81E-7 3E-7 1E-6

0 3E-91E-8 3E-81E-7 3E-7 1E-6

C

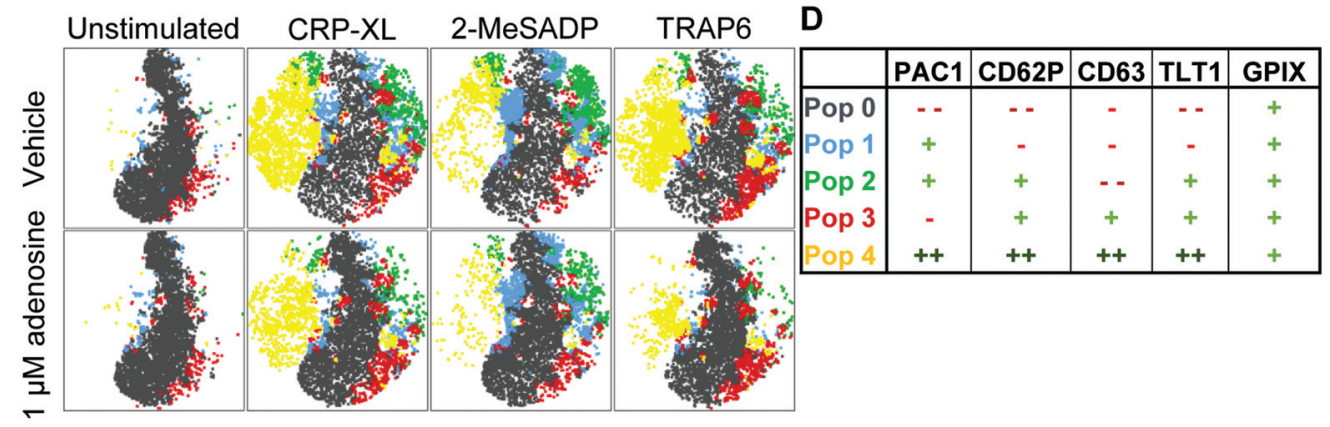

E
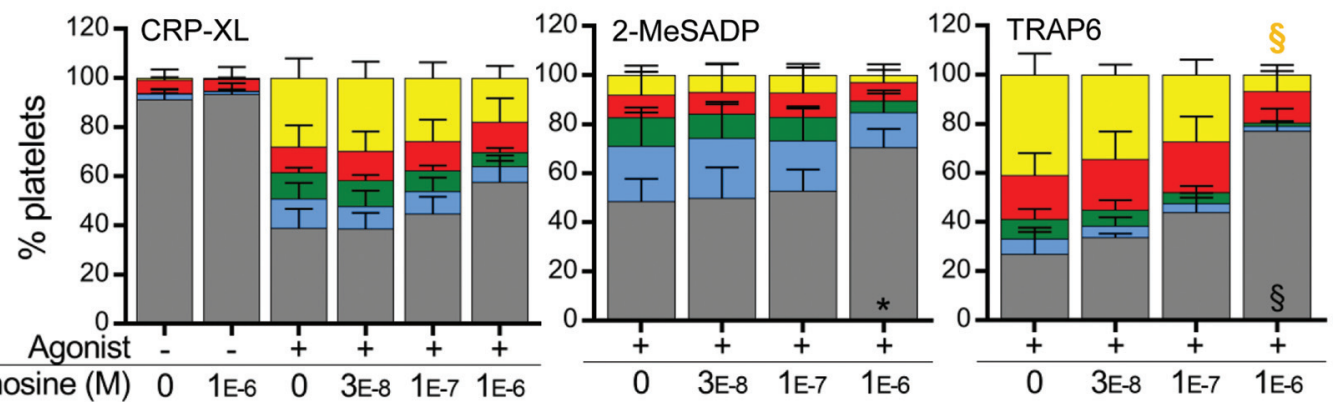

Fig. 4 Effect of adenosine priming on agonist-induced formation of platelet populations. Washed platelets were primed with adenosine before stimulation with suboptimal dose of CRP-XL, 2-MeSADP, or TRAP6 and stained with coloured PAC1 and anti-P-selectin, or additionally with antiCD63, anti-TLT1, and anti-GPIX mAbs for flow cytometry. (A) Flow cytometric events and corresponding histograms as overlaying two-colour plots: black = unstimulated (with $2 \%$ marker setting visible), purple =agonist (1.25 $\mu \mathrm{g} / \mathrm{mL}$ CRP-XL, $0.1 \mu \mathrm{M}$ ADP, and $7.5 \mu \mathrm{M}$ TRAP6), orange $=1$ $\mu \mathrm{M}$ adenosine plus agonist. (B) Distribution profile of platelet populations after priming with adenosine and indicated stimulation ( $2 \%$ threshold setting). (C) Representative tSNE plots of the vehicle- or $1 \mu \mathrm{M}$ adenosine-primed conditions. (D) Characterisation of the FlowSOM clusters (5 populations). (E) Distribution profile of platelet populations after priming with adenosine and indicated stimulation resulting from FlowSOM analysis. Mean $+\mathrm{SD}, n=4-5,{ }^{*} p<0.05$ compared with all other conditions, ${ }^{\ddagger} p<0.05$ compared with agonist only, $0.003,0.01,0.03$, and $0.1 \mu \mathrm{M}$ adenosine, ${ }^{\#} p<0.05$ compared with agonist only, $0.003,0.01$, and $0.03 \mu \mathrm{M}$ adenosine, ap $<0.05$ compared with agonist only, 0.003 and $0.01 \mu \mathrm{M}$ adenosine, ${ }^{*} p<0.05$ compared with agonist only and $0.003 \mu \mathrm{M}$ adenosine, ${ }^{\S} p<0.05$ compared with agonist only, 0.03 and $0.1 \mu \mathrm{M}$ adenosine; changes in population frequencies between conditions tested by chi-square multi-comparison test with false discovery rate correction. $\mathrm{mAb}$, monoclonal antibody; tSNE, t-distributed stochastic neighbor embedding. 
A

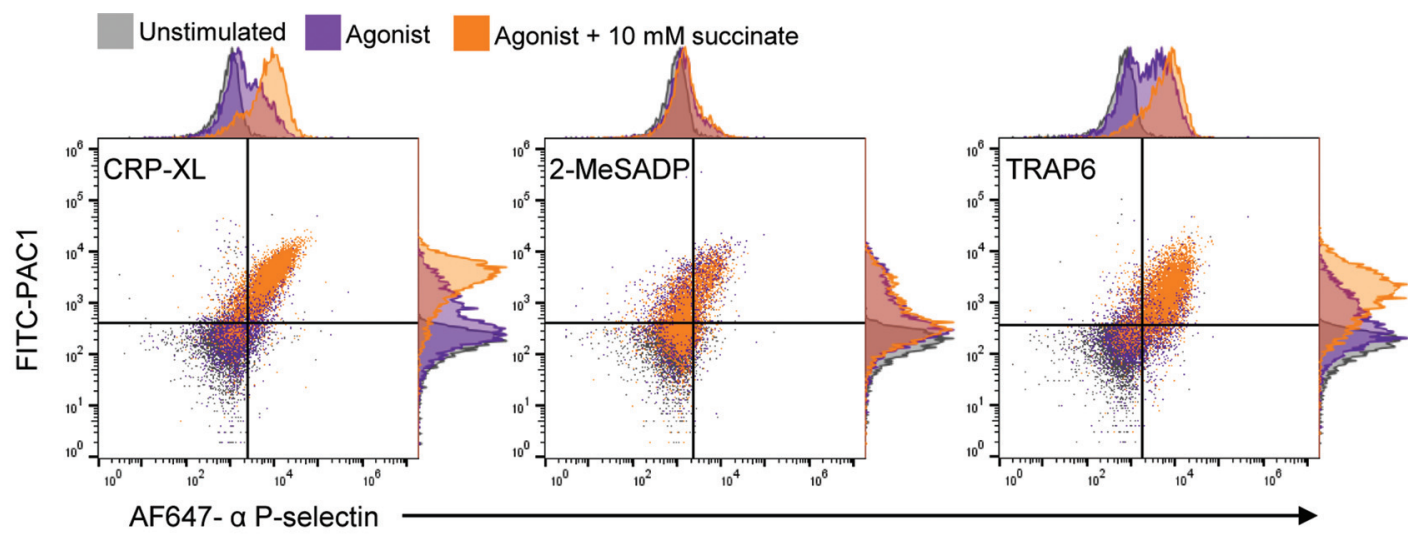

B

PAC1-/Psel- $\quad$ PAC1+/Psel- $\quad$ PAC1-/Psel+ PAC1+/Psel+

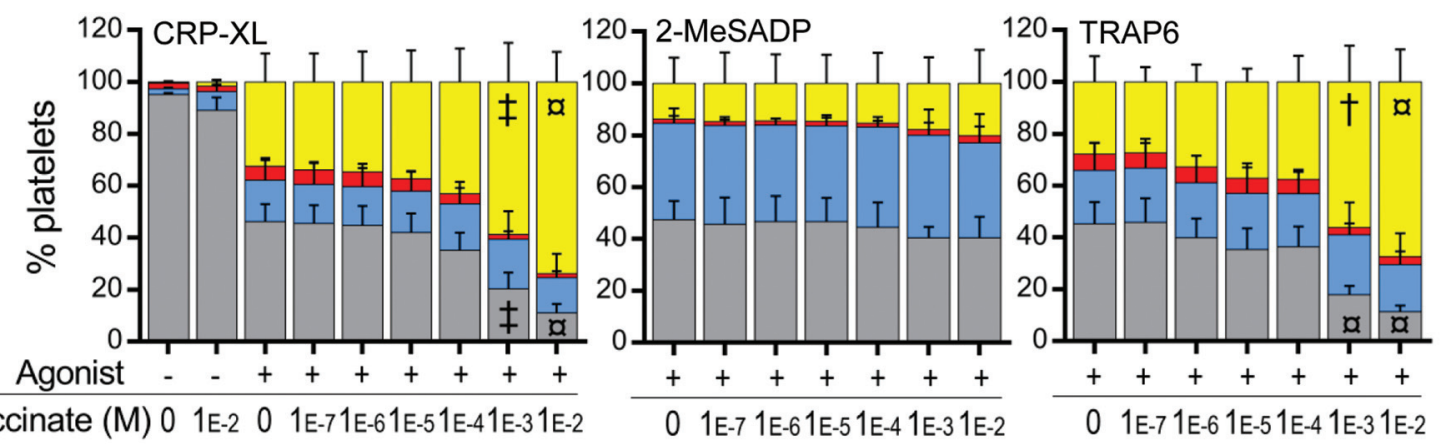

C
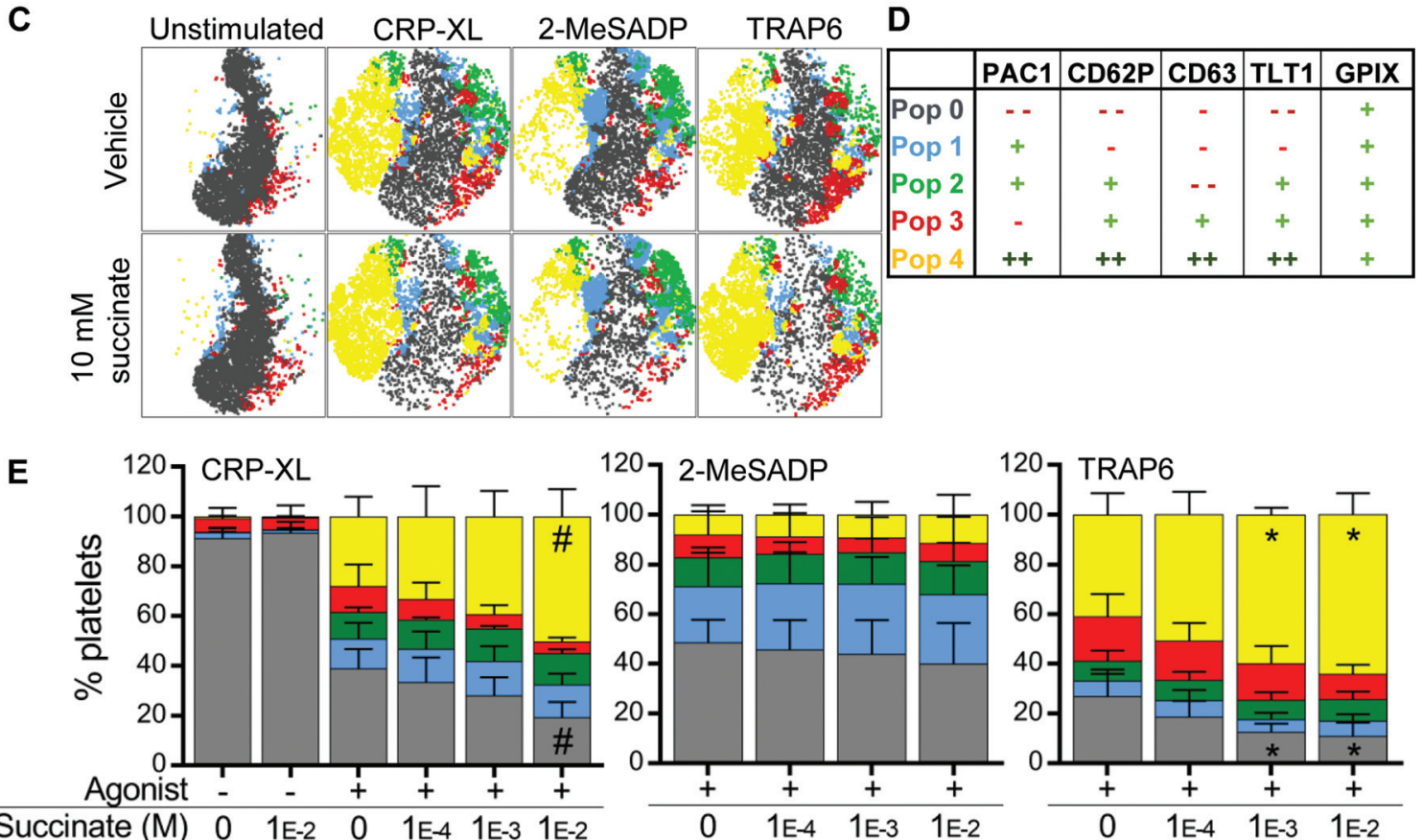

Fig. 5 Effect of succinate priming on agonist-induced formation of platelet populations. Platelets were primed with succinate before stimulation with a suboptimal dose of CRP-XL, 2-MeSADP, or TRAP6 and stained with coloured PAC1 and anti-P-selectin, or additionally with anti-CD63, antiTLT1, and anti-GPIX mAbs for flow cytometry. (A) Flow cytometric events and corresponding histograms as overlaying two-colour plots: black $=$ unstimulated (with $2 \%$ marker setting visible), purple $=$ agonist $(1.25 \mu \mathrm{g} / \mathrm{mL}$ CRP-XL, $0.1 \mu \mathrm{M}$ ADP, and $7.5 \mu \mathrm{M}$ TRAP6), orange $=$ succinate plus agonist. (B) Distribution profile of platelet populations after priming with succinate and indicated stimulation (2\% threshold setting). (C) Representative tSNE plots of the vehicle- or $10 \mathrm{mM}$ succinate-primed conditions. (D) Characterisation of the FlowSOM clusters (5 populations). (E) Distribution profile of platelet populations after priming with succinate and indicated stimulation resulting from FlowSOM analysis.

Mean $+S D, n=4-5, a p<0.05$ compared with agonist only, $0.1,1,10$, and $100 \mu \mathrm{M}$ succinate, ${ }^{\ddagger} p<0.05$ compared with agonist only, $0.1,1$, and $10 \mu \mathrm{M}$ succinate, ${ }^{\dagger} p<0.05$ compared with agonist only, 0.1 and $1 \mu \mathrm{M}$ succinate, ${ }^{\#} p<0.05$ compared with agonist only, ${ }^{*} p<0.05$ compared with all other conditions; changes in population frequencies between conditions tested by chi-square multi-comparison test with false discovery rate correction. SD, standard deviation; tSNE, t-distributed stochastic neighbor embedding. 
A

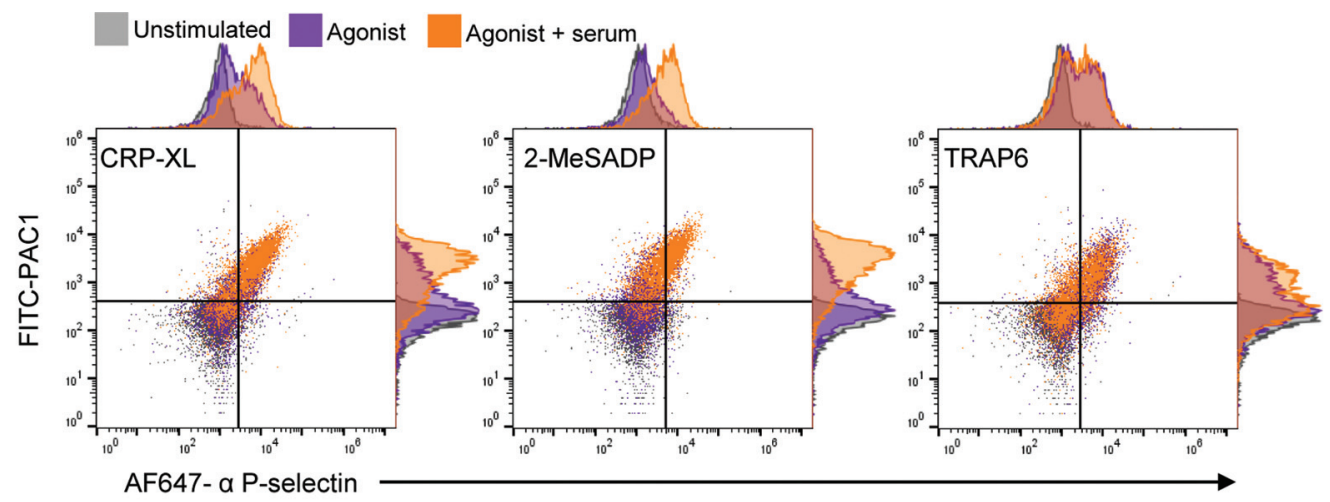

B

PAC1-/Psel- $\quad$ PAC1+/Psel- PAC1-/Psel+ PAC1+/Psel+

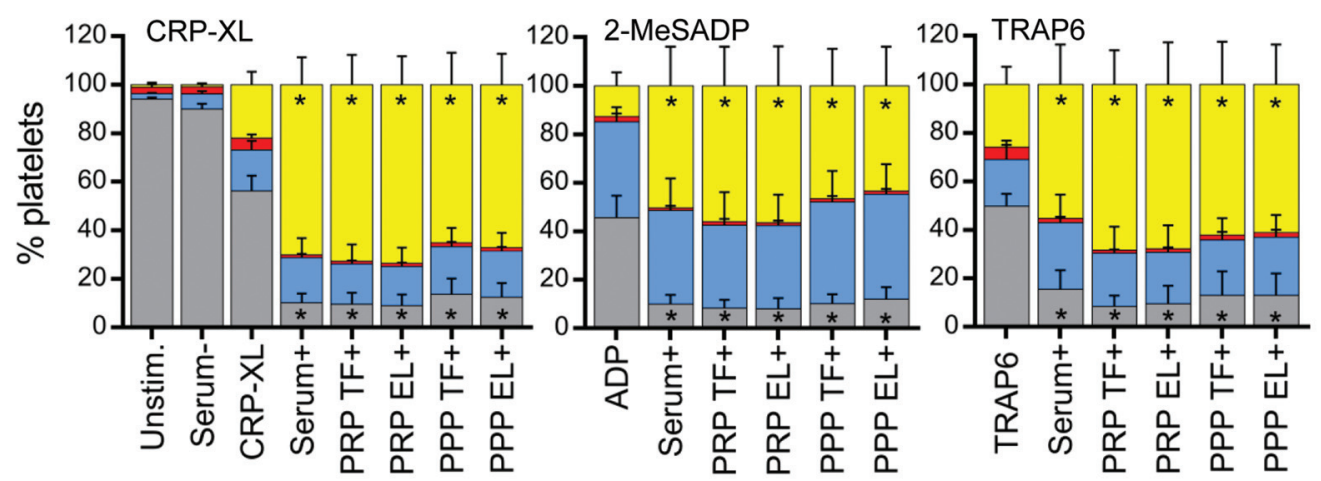

C

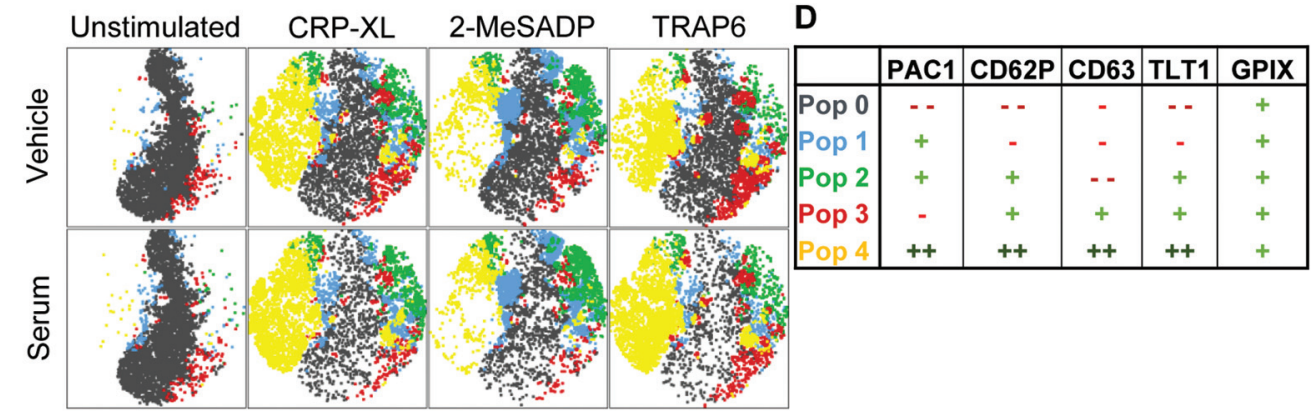

E

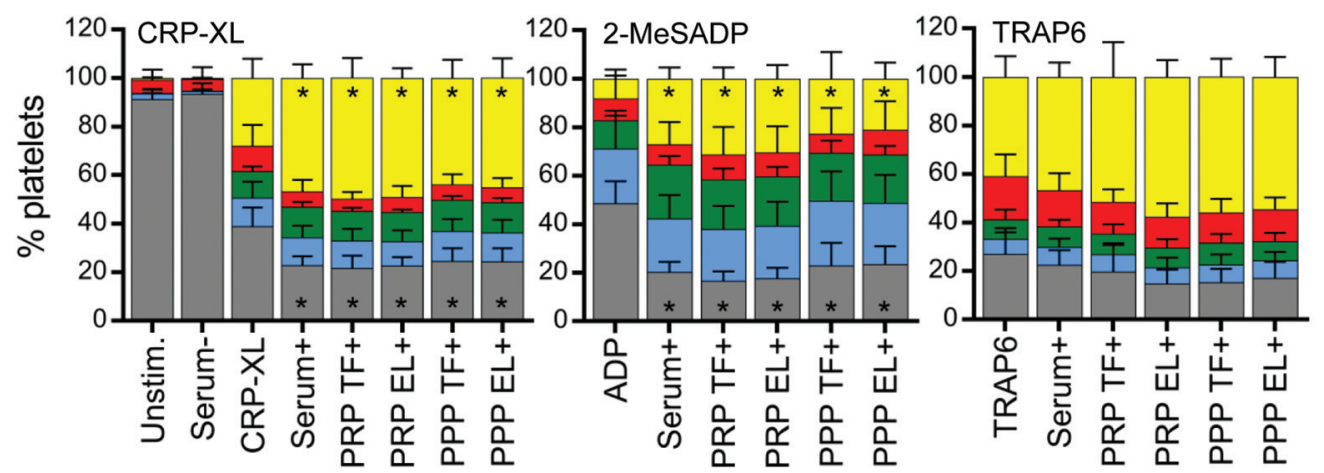

Fig. 6 Effect of platelet priming with serum or coagulated plasma on agonist-induced formation of platelet populations. Platelets were primed with serum or coagulated PRP or PPP; the plasmas were triggered with tissue factor (TF) or ellagic acid (EL), as indicated; thrombin was inactivated in all serum and plasma samples. For priming, undiluted serum or plasma was added, after which the platelets were stimulated with a suboptimal dose of CRP-XL, ADP, or TRAP6. The stimulated platelets were stained with fluorescently labelled PAC1 and anti-P-selectin, or additionally with anti-CD63, anti-TLT1, and anti-GPIX mAbs. (A) Flow cytometric events and corresponding histograms as overlaying two-colour plots: black = unstimulated, purple $=$ agonist $(1.25 \mu \mathrm{g} / \mathrm{mL}$ CRP-XL, $0.1 \mu \mathrm{M}$ ADP, and $7.5 \mu \mathrm{M}$ TRAP6), orange $=$ serum plus agonist. Quadrant gate was set for unstimulated sample of $2 \%$ positive for each fluorescent label. (B) Distribution profile of platelet populations after priming with serum or coagulated PRP or PPP, and stimulation with indicated agonist ( $2 \%$ threshold setting). (C) Representative tSNE plots of the vehicle- or serumprimed conditions. (D) Characterisation of the FlowSOM clusters (5 populations). (E) Distribution profile of platelet populations after priming with serum or coagulated plasma and indicated stimulation resulting from FlowSOM analysis. Mean $+S D, n=4-5,{ }^{*} p<0.05$ compared with all other conditions; changes in population frequencies between conditions tested by chi-square multi-comparison test with false discovery rate correction. PPP, platelet-poor plasma; PRP, platelet-rich plasma; SD, standard deviation; tSNE, t-distributed stochastic neighbor embedding. 
while the stimulus strength and the presence of primers mainly changed the populations of fully activated and resting platelets.

Detailed flow cytometric analysis indicated that, for establishing the platelet populations with integrin activation (PAC1 mAb binding) or secretion (P-selectin/CD62P expression), a choice needs to be made between identifying all platelets with (intermediate or high) surface activation markers (SED algorithm) or assessment of those platelets with $98 \%$ certain positivity for single or double activation markers ( $2 \%$ threshold settings in two colours). Regardless of the platelet agonist used, the former method gave $+7-20 \%$ positive events, due to incomplete histogram curve shifts. SED uses an improved normalisation and population estimation, thereby picking up small shifts in activation parameters. ${ }^{35}$ Accordingly, the SED algorithm appeared to be the most sensitive tool to assess intermediate platelet activation states. However, the algorithm cannot yet be used in multicolour flow cytometry, for which reason we applied the conventional $2 \%$ threshold marker settings and performed automated clustering analyses for identifying aggregating and/or secreting platelet populations.

Both analysis methods showed a typical agonist-specific distribution of platelet populations, largely independent of the agonist dose. The stronger agonists CRP-XL and TRAP6 showed with higher doses a substantial increase of the dual-positive $\left(\mathrm{PAC}^{+} / \mathrm{Psel}^{+}\right)$platelet fraction. With these agonists, minor platelet fractions with only integrin $\alpha_{\mathrm{IIb}} \beta_{3}$ activation or P-selectin expression were formed. Conversely, with 2-MeSADP (again dose-independently) the largest platelet fraction showed integrin activation without Pselectin expression. With all agonists, dose-dependently, the double-positive fraction increased at the expense of the double-negative fraction. Interestingly, clustering analyses of multicolour data disclosed for all agonists a platelet population characterised by complete secretion of $\alpha-$ and dense granules/lysosomes, but inactive integrins. It is unlikely that inactivation of previously activated integrins has occurred, since the PAC1 antibody prevents integrin closure. $^{38}$ In addition, a platelet population with activated integrins and only $\alpha$-granule secretion, but no dense granule release, was identified. To our knowledge, this study is the first one to clearly identify this population. It was suggested before that dense granular release is the fastest secretory route, whereas $\alpha$-granule and lysosomal secretion are slower. ${ }^{39,40}$ Little is known so far on the secretory heterogeneity within one individual's platelet pool.

Priming with adenosine resulted in a decreased platelet activation status with all three agonists tested. Typically, the negative priming effect of $1 \mu \mathrm{M}$ adenosine was most pronounced after stimulation with ADP or TRAP6, compared with CRP-XL. Positive priming of platelets with succinate did not show the opposite effect of negative priming with adenosine. ADP-induced platelet activation was less influenced by succinate treatment than platelet activation after CRP-XL or TRAP6 stimulation. Also, in an earlier study, succinate only limitedly induced P-selectin expression at higher ADP doses. ${ }^{41}$ The finding that coagulated plasmas (from PRP or PPP) exerted similar effects as serum indicated that the modulating agent(s) are not of blood cellular origin. The exact compound(s) responsible for this priming need to be further elucidated. Altogether, the present results suggest that glycoprotein VI (GPVI)induced platelet signalling is more sensitive to a decrease in the priming-dependent activation threshold (succinate or coagulated plasma) than to an increase in this threshold (adenosine). Interestingly, the primers did only affect the populations of fully activated and resting platelets, while the other populations (aggregating-only, secreting-only, aggregating plus $\alpha$-granule secretion) remained unchanged. This suggests that a subset of platelets exerts a fixed, constant response to different types of stimuli. Differences in results for the aggregating and secreting platelet populations between the two-colour and the multicolour flow cytometry might be explained by the arbitrary $2 \%$ marker setting compared with the automated clustering algorithms, the latter being more sensitive to intermediately activated platelets.

The type and level of priming factors that either reduce (adenosine) or enhance (succinate, coagulation products) platelet responsiveness will determine the contributing role of platelets in pathological conditions, such as ischaemic heart disease. Although evidence suggests that the adenosine level is increased in these patients, its inhibiting effect on platelets might be attenuated by the relatively low level of $\mathrm{A}_{2 \mathrm{~A}}$ receptors on cardiovascular cells. ${ }^{10}$ Thereby, the plateletstimulating role of succinate may dominate and can antagonise the effects of antiplatelet drugs. ${ }^{41}$ This antagonising result on the effects of antiplatelet drugs could also be observed for the presence of coagulation products (data not shown).

The identification of distinct platelet populations with specific functions will provide mechanistical insights into the role of platelets in the pathogenesis of disease states, even in processes beyond thrombosis and haemostasis (e.g., inflammation, cancer, and immunity). Furthermore, studies by Dale and colleagues presented evidence that the population of coated platelets, characterised by the surface exposure of phosphatidylserine and retention of procoagulant proteins, was elevated in patients with ischaemic stroke and improved stroke prediction. ${ }^{42-45}$ These studies indicate that platelet subpopulations might serve as a biomarker for disease and efficacy of treatment in the future. The identification and isolation of specific platelet subpopulations could also be beneficial in transfusion practice for optimising the effect of platelet concentrates in different clinical settings.

Based on the results of this study, we advocate the further development and use of analysis algorithms to accurately define the activation status of platelets and thereby the appearance of diverse platelet populations. This will be especially important when assessing the enhancing and/or 
suppressing effects of blood-borne modulators, prominent in different disease states, on platelet activity.

\section{What is known about this topic?}

- There is heterogeneity in platelet composition and function.

- Priming of platelets by bioactive molecules in the circulation alters the activation threshold.

- Multicolour flow cytometry can be used to determine the platelet activation status.

\section{What does this paper add?}

- Multicolour flow cytometry and clustering analyses identify platelet populations following platelet activation and priming.

- The type of agonist determines the typical distribution of platelet populations, while priming substances only affect the populations of fully activated and resting platelets.

- GPVI-induced platelet signalling is more sensitive to a decrease in the priming-dependent activation threshold (succinate or coagulated plasma) than to an increase in this threshold (adenosine).

\section{Funding}

This work is supported by the Landsteiner Foundation for Blood Transfusion Research Grant No. 1711. C.C.F.M.J.B. received funding from The Dutch Heart Foundation (2020T020) and the START-Program of the Faculty of Medicine at the RWTH Aachen University (105/20). I.D. $\mathrm{S}$. is supported by a joint PhD scholarship of Maastricht and Reading Universities, and by the European Union's Horizon 2020 research and innovation program under the Marie Sklodowska-Curie grant agreement No. 766118.

Conflict of Interest

None declared.

\section{Acknowledgments}

We thank Dr. Sofie van Gassen for assisting with the FlowSOM analyses and Alejandro Pallerés Robles for assisting with the statistical analyses.

\section{References}

1 Baaten CCFMJ, Ten Cate H, van der Meijden PEJ, Heemskerk JWM. Platelet populations and priming in hematological diseases. Blood Rev 2017;31(06):389-399

2 van der Meijden PEJ, Heemskerk JWM. Platelet biology and functions: new concepts and clinical perspectives. Nat Rev Cardiol 2019;16(03):166-179

3 Swieringa F, Kuijpers MJ, Heemskerk JW, van der Meijden PE. Targeting platelet receptor function in thrombus formation: the risk of bleeding. Blood Rev 2014;28(01):9-21

4 Gariboldi V, Vairo D, Guieu R, et al. Expressions of adenosine $A_{2 A}$ receptors in coronary arteries and peripheral blood mononuclear cells are correlated in coronary artery disease patients. Int J Cardiol 2017;230:427-431

5 Marlinge M, Vairo D, Marolda V, et al. Rapid measurement of adenosine concentration in human blood using FixedPotential amperometry: comparison with mass spectrometry and highperformance liquid chromatography. J Anal Bioanal Tech 2017; 2017:1-5

6 Funaya H, Kitakaze M, Node K, Minamino T, Komamura K, Hori M. Plasma adenosine levels increase in patients with chronic heart failure. Circulation 1997;95(06):1363-1365

7 Aguiar CJ, Rocha-Franco JA, Sousa PA, et al. Succinate causes pathological cardiomyocyte hypertrophy through GPR91 activation. Cell Commun Signal 2014;12:78

8 Eltzschig HK, Ibla JC, Furuta GT, et al. Coordinated adenine nucleotide phosphohydrolysis and nucleoside signaling in posthypoxic endothelium: role of ectonucleotidases and adenosine A2B receptors. J Exp Med 2003;198(05):783-796

9 Eckle T, Krahn T, Grenz A, et al. Cardioprotection by ecto-5'nucleotidase (CD73) and A2B adenosine receptors. Circulation 2007;115(12):1581-1590

10 Paganelli F, Gaudry M, Ruf J, et al. Recent advances in the role of the adenosinergic system in coronary artery disease. Cardiovasc Res 2021;117(05):1284-1294

11 Johnston-Cox HA, Ravid K. Adenosine and blood platelets. Purinergic Signal 2011;7(03):357-365

12 Fuentes E, Pereira J, Mezzano D, Alarcón M, Caballero J, Palomo I. Inhibition of platelet activation and thrombus formation by adenosine and inosine: studies on their relative contribution and molecular modeling. PLoS One 2014;9(11):e112741

13 Löfgren L, Pehrsson S, Hägglund G, Tjellström H, Nylander S. Accurate measurement of endogenous adenosine in human blood. PLoS One 2018;13(10):e0205707

14 Martin C, Leone M, Viviand X, Ayem ML, Guieu R. High adenosine plasma concentration as a prognostic index for outcome in patients with septic shock. Crit Care Med 2000;28(09):3198-3202

15 Gaudry M, Vairo D, Marlinge M, et al. Adenosine and its receptors: an expected tool for the diagnosis and treatment of coronary artery and ischemic heart diseases. Int J Mol Sci 2020;21(15):E5321

16 Mills E, O'Neill LA. Succinate: a metabolic signal in inflammation. Trends Cell Biol 2014;24(05):313-320

17 Sanborn T, Gavin W, Berkowitz S, Perille T, Lesch M. Augmented conversion of aspartate and glutamate to succinate during anoxia in rabbit heart. Am J Physiol 1979;237(05):H535-H541

18 Taegtmeyer H. Metabolic responses to cardiac hypoxia. Increased production of succinate by rabbit papillary muscles. Circ Res 1978;43(05):808-815

19 Kakinuma Y, Matsubara T, Hashimoto T, Sakamoto N. Myocardial metabolic markers of total ischemia in vitro. Nagoya J Med Sci 1994;57(1-4):35-42

20 Hakak Y, Lehmann-Bruinsma K, Phillips S, et al. The role of the GPR91 ligand succinate in hematopoiesis. J Leukoc Biol 2009;85 (05):837-843

21 Grimolizzi F, Arranz L. Multiple faces of succinate beyond metabolism in blood. Haematologica 2018;103(10):1586-1592

22 Macaulay IC, Tijssen MR, Thijssen-Timmer DC, et al. Comparative gene expression profiling of in vitro differentiated megakaryocytes and erythroblasts identifies novel activatory and inhibitory platelet membrane proteins. Blood 2007;109(08):3260-3269

23 Loeffen R, Spronk HM, ten Cate H. The impact of blood coagulability on atherosclerosis and cardiovascular disease. J Thromb Haemost 2012;10(07):1207-1216

24 Gerotziafas GT, Zografos T, Pantos I, et al. Prospective assessment of biomarkers of hypercoagulability for the identification of patients with severe coronary artery disease. The ROADMAP-CAD study. Clin Appl Thromb Hemost 2020;26:1076029620964590

25 White TC, Berny MA, Tucker EI, et al. Protein C supports platelet binding and activation under flow: role of glycoprotein $\mathrm{Ib}$ and apolipoprotein E receptor 2.J Thromb Haemost 2008;6(06):995-1002 
26 Magwenzi SG, Ajjan RA, Standeven KF, Parapia LA, Naseem KM Factor XIII supports platelet activation and enhances thrombus formation by matrix proteins under flow conditions. J Thromb Haemost 2011;9(04):820-833

27 Maecker HT, McCoy JP Jr, Amos M, et al; FOCIS Human Immunophenotyping Consortium. A model for harmonizing flow cytometry in clinical trials. Nat Immunol 2010;11(11): 975-978

28 Pitoiset F, Cassard L, El Soufi K, et al. Deep phenotyping of immune cell populations by optimized and standardized flow cytometry analyses. Cytometry A 2018;93(08):793-802

29 Baaten CCFMJ, Moenen FCJI, Henskens YMC, et al. Impaired mitochondrial activity explains platelet dysfunction in thrombocytopenic cancer patients undergoing chemotherapy. Haematologica 2018;103(09):1557-1567

30 Södergren AL, Ramström S. Platelet subpopulations remain despite strong dual agonist stimulation and can be characterised using a novel six-colour flow cytometry protocol. Sci Rep 2018;8 (01):1441

31 Blair TA, Moore SF, Hers I. Circulating primers enhance platelet function and induce resistance to antiplatelet therapy. J Thromb Haemost 2015;13(08):1479-1493

32 Schmitz G, Rothe G, Ruf A, et al. European Working Group on Clinical Cell Analysis: consensus protocol for the flow cytometric characterisation of platelet function. Thromb Haemost 1998;79 (05):885-896

33 Waller AK, Lantos L, Sammut A, et al. Flow cytometry for nearpatient testing in premature neonates reveals variation in platelet function: a novel approach to guide platelet transfusion. Pediatr Res 2019;85(06):874-884

34 Gilio K, Munnix IC, Mangin P, et al. Non-redundant roles of phosphoinositide 3-kinase isoforms alpha and beta in glycoprotein VI-induced platelet signaling and thrombus formation. J Biol Chem 2009;284(49):33750-33762
35 Bagwell B. Super enhanced D-value method (possed). Accessed September 5, 2021 at: http://www.vsh.com/Publication/SuperEnhancedDValue.pdf

36 Monaco G, Chen H, Poidinger M, Chen J, de Magalhães JP, Larbi A. flowAI: automatic and interactive anomaly discerning tools for flow cytometry data. Bioinformatics 2016;32(16):2473-2480

37 Van Gassen S, Callebaut B, Van Helden MJ, et al. FlowSOM: using self-organizing maps for visualization and interpretation of cytometry data. Cytometry A 2015;87(07):636-645

38 Mattheij NJ, Gilio K, van Kruchten R, et al. Dual mechanism of integrin $\alpha$ IIb $\beta 3$ closure in procoagulant platelets. J Biol Chem 2013;288(19):13325-13336

39 Eckly A, Rinckel JY, Proamer F, et al. Respective contributions of single and compound granule fusion to secretion by activated platelets. Blood 2016;128(21):2538-2549

40 Jonnalagadda D, Izu LT, Whiteheart SW. Platelet secretion is kinetically heterogeneous in an agonist-responsive manner. Blood 2012;120(26):5209-5216

41 Spath B, Hansen A, Bokemeyer C, Langer F. Succinate reverses invitro platelet inhibition by acetylsalicylic acid and P2Y receptor antagonists. Platelets 2012;23(01):60-68

42 Prodan CI, Joseph PM, Vincent AS, Dale GL. Coated-platelets in ischemic stroke: differences between lacunar and cortical stroke. J Thromb Haemost 2008;6(04):609-614

43 Prodan CI, Stoner JA, Cowan LD, Dale GL. Higher coated-platelet levels are associated with stroke recurrence following nonlacunar brain infarction. J Cereb Blood Flow Metab 2013;33(02):287-292

44 Kirkpatrick AC, Stoner JA, Dale GL, Prodan CI. Elevated coatedplatelets in symptomatic large-artery stenosis patients are associated with early stroke recurrence. Platelets 2014;25(02):93-96

45 Kirkpatrick AC, Tafur AJ, Vincent AS, Dale GL, Prodan CI. Coatedplatelets improve prediction of stroke and transient ischemic attack in asymptomatic internal carotid artery stenosis. Stroke 2014;45(10):2995-3001 